\title{
Extracción y caracterización de pectina de mango de azúcar (Mangifera indica L.)
}

\section{Extraction and characterization of pectin from sugar mango(Mangifera indica $\mathrm{L}$. )}

\author{
Genisberto E. Barreto', Amparo L. Púa², Dilan D. De Alba ${ }^{3}$, María M. Pión ${ }^{3}$ \\ Recibido para publicación: Enero 18 de 2017 - Aceptado para publicación: Abril 18 de 2017
}

\begin{abstract}
RESUMEN
La pectina es un producto tecnológicamente funcional por sus propiedades reológicas. En Colombia en el año 2010 se importaron aproximadamente 195 toneladas de pectina y su demanda sigue en aumento. El objetivo del estudio fue extraer y caracterizar la pectina obtenida de la cáscara de mango de azúcar maduro. El aislamiento del material se realizó mediante hidrolisis ácida, con ácido clorhídrico 0,5 N y alcohol etílico al 96\% para su precipitación y purificación. Para la extracción se utilizó un arreglo factorial $3^{2}$, evaluando los factores $\mathrm{pH}$ y temperatura, con los niveles $1,2,3$ y $80,90,100{ }^{\circ} \mathrm{C}$ respectivamente, estableciendo como variable fija un tiempo de 60 minutos. Se dispuso de 27 unidades experimentales equivalentes a 550 g de cáscara de mango de azúcar maduro seca y molida. Los mejores resultados para la obtención de la pectina fueron $\mathrm{pH} 1$ y temperatura $100{ }^{\circ} \mathrm{C}$, reportando un rendimiento de $15,257 \pm 0,04 \%$. La pectina obtenida presentó las siguientes características: humedad 4,510 $\pm 0,80 \%$, cenizas $1,351 \pm 0,07 \%$, alcalinidad de las cenizas 1,711 $\pm 1,13 \%$, acidez libre 0,859 $\pm 0,01 \mathrm{mEq}$ de carboxilo libre/g, peso equivalente 2326,420 $\pm 54,11$ $\mathrm{mg} / \mathrm{mEq}$, contenido de metoxilo $11,801 \pm 0,03 \%$, grado de esterificación 81,688 $\pm 024 \%$, contenido de ácido anhidrogalacturónico $82,380 \pm 0,17 \%$, el análisis de espectrofotometría infrarrojo, mostró los picos de grupos funcionales, característicos de la pectina obtenida y su similitud frente a una pectina estándar. El material estudiado es fuente potencial de pectina de calidad.
\end{abstract}

Palabras clave: Ácido anhidrogalacturónico, espectro infrarrojo, esterificación, hidrólisis ácida, metoxilo.

\begin{abstract}
Pectin is a functional technology product by her rheological characteristics. In 2010 in Colombia, there were imported approximately 195 tons and its demand is increasing. The main goal of this research was to extract and characterize the pectin from ripe sugar mango peel. The isolation of pectin material was accomplished by acid hydrolysis, with hydrochloric acid to $0.5 \mathrm{~N}$ and ethyl alcohol to $96 \%$ for precipitation and purification. For the extraction of pectin factorial arrangement 32 was used where the two factors evaluated were: $\mathrm{pH}$ and temperature, which took the levels 1, 2, 3 and 80, 90, 100 respectively, in a constant time of 60 minutes. There were 27 experimental units equivalent to $550 \mathrm{~g}$ of ripe, dry and ground sugar mango peel. The results were evaluated and it was stated that ideal variables $(\mathrm{pH} 1$ and temperature $100{ }^{\circ} \mathrm{C}$ ) allow to extract major quantity of pectin, bringing a performance of $15.257 \pm 0.04 \%$. The obtained pectin presented the following characteristics: humidity content $(4.510 \pm 0.80 \%)$, ashes content $(1.351 \pm 0.07 \%)$, alkalinity of the ashes $(1.711 \pm 1.13 \%)$, free acidity $(0.859 \pm 0.01 \mathrm{mEq}$ free / g carboxyl), equivalent weight $(2326.420 \pm 54.11 \mathrm{mg}$ / $\mathrm{mEq})$, methoxyl $(11,801 \pm 0.03 \%)$, degree of esterification $(81.688 \pm 024 \%)$, anhydrous acid content $(82.380 \pm 0.17 \%)$ and the analysis by infrared spectrophotometry, showed the peaks of functional groups, typical of the obtained pectin and its similarity opposite to a standard pectin. The material studied is a potential source of quality pectin.
\end{abstract}

Keywords: Acid hydrolysis, anhydrous galacturonic acid, degree of esterification, infrared spectrophotometry, methoxy.

\footnotetext{
${ }^{1}$ Especialista en Química de Alimentos, Docente de Tiempo Completo de La Universidad del Atlántico.

$2^{2 *}$ M.Sc. Docente de Tiempo Completo de La Universidad del Atlántico, Barranquilla, Colombia.

${ }^{3}$ Estudiantes de Ingeniería Agroindustrial, Universidad del Atlántico, Barranquilla, Colombia. *Km 7 Antigua carretera a Puerto Colombia, celular: 3145023185, genisbertobarreto@mail.uniatlantico.edu.co
} 


\section{INTRODUCCIÓN}

Las pectinas son polisacáridos que se componen principalmente de unidades de ácido galacturónico unidos por enlaces glucosídicos $\alpha$ 1-4, son sustancias blandas amorfas que forman en agua una solución viscosa; combinadas en proporciones adecuadas con azúcar y ácido, forman una sustancia gelatinosa utilizada como espesante, Fennema (1993).

La pectina es utilizada por la industria alimentaria como gelificante, espesante, estabilizante y emulsificante; igualmente por la industria farmacéutica y el área de biotecnología para la formulación de fármacos y cosméticos, Sriamornsak, (2003); Mamani et al. (2012).

El elevado consumo de fibra dietaria como la pectina, produce efectos favorables en la salud humana por sus propiedades anticancerígenas, hipoglicemiantes e hipocolesterolémicas, Martínez et al. (2011), también disminuye el riesgo de sufrir hipertensión, obesidad y ciertos desórdenes gastrointestinales, Falcon et al. (2011).

Teniendo en cuenta la necesidad de la industria colombiana y que la totalidad de la pectina es importada como materias pécticas, pectinatos y pectatos, según las necesidades de los productos, García y Penagos (2011) se requiere encontrar fuentes potenciales de este material, que puede generarse a partir del aprovechamiento de frutas tropicales. En Colombia y específicamente en la región Caribe existe variedad de frutas, dentro de ellas el mango de azúcar; el mango colombiano tiene una gran demanda en el país y su consumo abarca más del 95 \% de la producción nacional, Asohofrucol, Ministerio de Agricultura y Desarrollo Rural, Corpoica (2009).

El mango es rico en agua, azúcares, fibra, minerales, vitaminas especialmente vitaminas $\mathrm{C}$ y A, ácidos orgánicos como el málico, palmítico, p-cumárico y mirístico, Dussan et al. (2014). El aprovechamiento industrial del mango se ha basado comúnmente en la obtención de la pulpa, su parte comestible representa de un 33\% a un $85 \%$ aproximadamente de la fruta fresca, mientras que la cáscara y la semilla de un $7 \%$ a $24 \%$ y de $9 \%$ a $40 \%$ respectivamente, Campos y Guerrero (2014); aparte de la obtención de pulpa también se obtienen productos secundarios que suman del $35 \%$ al $60 \%$ del peso total de la fruta, Berardini et al. (2005).

En general son muchos los usos del mango, y se le atribuyen también propiedades laxantes, diuréticas y vigorizantes, al jugo de mango verde asado en cenizas calientes se le atribuyen propiedades refrigerantes, se asegura que contribuye al tratamiento del cólera y la peste; la quema de las hojas verdes ayuda a las inflamaciones de la garganta, Medina (1981); la corteza tiene mangíferina, sustancia astringente, usada para combatir el reumatismo y la difteria, en países como La India; los extractos a base de frutos inmaduros, corteza, tallos y hojas presentan actividad antibiótica; la cocción de las hojas se ha usado para tratar la diarrea, la fiebre, el dolor de pecho, la diabetes, la hipertensión, Morton (1987).

El procesamiento del mango de azúcar (pulpa, néctar, jugo) genera alteraciones ambientales negativas por la gran cantidad de materiales sólidos que se eliminan; actualmente estos desechos son reutilizados para obtención de compostaje, alimento para ganado o generación de energía, Koubala et al. (2008); otra alternativa de aprovechamiento de los residuos sería la obtención de pectinas, lo que conduce a los siguientes beneficios para el mercado nacional: desarrollar una actividad económica que ayudaría a reemplazar un producto importado, contribuyendo al desarrollo económico y tecnológico del país; proporcionarle valor agregado a los cultivos, generando en los agricultores valoración de los recursos propios; ampliar conocimientos relacionados con el mango de azúcar; aprovechar los cultivos frutales con fines tecnológicos, ofreciendo una 
alternativa diferente al consumo en fresco y a la medicina popular, Montaña (2016).

El objetivo del presente estudio fue extraer y caracterizar la pectina de la cáscara del mango de azúcar maduro.

\section{MATERIALES Y MÉTODOS}

Se seleccionó una muestra aleatoria de mango de azúcar maduro proveniente de Sitio Nuevo, Magdalena (Colombia), con las características mínimas exigidas por la Norma Técnica Colombiana 5139, ICONTEC (2002); se determinó la concentración de sólidos solubles, acidez titulable e índice de madurez.

La concentración de sólidos solubles se determinó por el método refractométrico, empleando refractómetro marca Abbe, el resultado se expresó en grados Brix, Gamboa (2009).

La acidez titulable se obtuvo a partir del método volumétrico, Gamboa (2009), el resultado se expresó como porcentaje de ácido málico. El índice de madurez se obtuvo de la relación entre los grados Brix obtenidos y la acidez titulable, García et al. (2010). La fruta fue despulpada, las cáscaras se sometieron a lavados sucesivos con agua potable y destilada, para eliminar impurezas o suciedad presente en la superficie. A este material se le extrajo la mayor cantidad de agua mediante una tela de lona y se trituró en una picadora marca Imusa, para aumentar el área de contacto y así facilitar el proceso de extracción de la pectina.

En la inactivación enzimática se utilizó aproximadamente $1 \mathrm{~kg}$ de materia prima, se sometió a tratamiento térmico con agua a 95-98 ${ }^{\circ} \mathrm{C}$ durante 15 min, la mezcla se dejó decantar y se descartó el agua residual. Se establecieron los parámetros óptimos de $\mathrm{pH}$ y temperatura para la hidrolisis ácida, mediante un arreglo factorial $3^{2}$, la pectina obtenida se secó en una estufa de desecación marca Memmert a $50{ }^{\circ} \mathrm{C}$ hasta que alcanzo un peso constante, luego la pectina fue molida, para su posterior caracterización.

A las pectinas se le realizaron las siguientes determinaciones por triplicado: humedad, mediante una balanza halógeno marca OHAUS MB35 halogen, Muñoz (2011); cenizas por el método de ignición utilizando la mufla Terrígeno, Arellano y Hernández (2013); alcalinidad de las cenizas, se calculó en termino de carbonato, León y Riveros (2014); para el peso equivalente, acidez libre y grupos metoxilo, se empleó el método descrito por Ferreira (2007); el porcentaje de esterificación por Baltazar et al. (2013); para la determinación de ácido anhidro galaracturónico, se relacionó la masa de la muestra el contenido de acidez libre, la alcalinidad de las cenizas y el contenido de metoxilo, Ferreira (2007); hierro y calcio por Suzanne (2010).

Para la cuantificación del hierro y calcio, se llevó una muestra de la solución preparada anteriormente a un espectrofotómetro de absorción atómica marca Spectroquant Nova 600.

Para la determinación la viscosidad se elaboró una mermelada, cuyas materias primas utilizadas fueron: pulpa de fresa, azúcar de caña, ácido cítrico y pectina de cáscara de mango, el proceso de elaboración finalizó hasta que la mezcla alcanzó los $65{ }^{\circ}$ Brix, medidos con un refractómetro marca Abbe, seguidamente se envasó, enfrió y almacenó, Flórez (2013); luego la muestra de mermelada climatizada se llevó al viscosímetro de Brookfield digital empleando una velocidad de agitaciones de 100 rpm, temperatura de la muestra de $25{ }^{\circ} \mathrm{C}$ y tiempo de agitación de un minuto, Suzanne (2010), se realizó la lectura directa en el equipo, en unidades de Centipoise (cps).

Los espectros infrarrojos de las pectinas extraídas, se obtuvieron con el equipo Perkin-Elmer 1600 series FTIR Modelo 1605, Stuart (2005); los resultados del rendimiento obtenidos, se so- 
metieron a un análisis estadístico mediante el programa IBM SPSS Statistics 20, con el objetivo de determinar si las variables eran estadísticamente significativas con un $95 \%$ de confianza.

Los resultados de la caracterización fueron promediados y luego se sometieron a una comparación para determinar las diferencias o similitudes frente a la media de tres pectinas estándar.

\section{RESULTADOS Y DISCUSIÓN}

Los resultados promediados del porcentaje de acidez del fruto fueron 0,1389 , concentración de sólidos solubles $18{ }^{\circ}$ Brix y el índice de madurez 129,502, lo que infiere que el estado de madurez, produjo disminución de la acidez titulable y aumento de la concentración de sólidos solubles, característico de las frutas climatéricas, Montaña (2016). La tabla 1 corresponde al rendimiento de la pectina obtenido en diferentes condiciones experimentales y en esta se deduce que los $\mathrm{pH}$

Tabla 1. Porcentaje de rendimiento de la pectina en diferentes condiciones experimentales.

\begin{tabular}{|c|c|c|c|c|}
\hline Tratamiento & $\begin{array}{c}\text { TIEMPO } \\
\text { (Minutos) }\end{array}$ & $\mathrm{pH}$ & $\begin{array}{l}\text { TEMPERATU- } \\
\text { RA }\left({ }^{\circ} \mathrm{C}\right)\end{array}$ & Rendimiento \\
\hline 1 & 60 & 1 & 80 & 14,04 \\
\hline 2 & 60 & 2 & 80 & 9,63 \\
\hline 3 & 60 & 3 & 80 & 8,38 \\
\hline 4 & 60 & 1 & 90 & 14,48 \\
\hline 5 & 60 & 2 & 90 & 13,88 \\
\hline 6 & 60 & 3 & 90 & 13,42 \\
\hline 7 & 60 & 1 & 100 & 15,23 \\
\hline 8 & 60 & 2 & 100 & 13,67 \\
\hline 9 & 60 & 3 & 100 & 13,16 \\
\hline 10 & 60 & 1 & 80 & 14,17 \\
\hline 11 & 60 & 2 & 80 & 9,60 \\
\hline 12 & 60 & 3 & 80 & 9,08 \\
\hline 13 & 60 & 1 & 90 & 14,60 \\
\hline 14 & 60 & 2 & 90 & 12,93 \\
\hline 15 & 60 & 3 & 90 & 12,32 \\
\hline 16 & 60 & 1 & 100 & 15,31 \\
\hline 17 & 60 & 2 & 100 & 14,48 \\
\hline 18 & 60 & 3 & 100 & 13,18 \\
\hline 19 & 60 & 1 & 80 & 13,99 \\
\hline 20 & 60 & 2 & 80 & 9,67 \\
\hline 21 & 60 & 3 & 80 & 8,38 \\
\hline 22 & 60 & 1 & 90 & 14,49 \\
\hline 23 & 60 & 2 & 90 & 13,88 \\
\hline 24 & 60 & 3 & 90 & 13,41 \\
\hline 25 & 60 & 1 & 100 & 15,23 \\
\hline 26 & 60 & 2 & 100 & 13,67 \\
\hline 27 & 60 & 3 & 100 & 13,16 \\
\hline MEDIA & & & & $12,866 \pm 2,17$ \\
\hline
\end{tabular}

bajos y las temperaturas altas, proporcionan mayor rendimiento al proceso de extracción, estos resultados coinciden con los de Addosio et al. (2005), donde también se empleó como agente secuestrante el $\mathrm{HCl}$, lo que se puede relacionar con el estado de madurez del fruto, ya que en estado maduro, la pectina es degradada de forma natural y presenta una proporción pequeña de grupos carboxilos esterificados.

La tabla 2 expresa que el $\mathrm{pH}$ y la temperatura son estadísticamente significativos, con un valor p que es menor a 0,05, con un $95 \%$ de confiabilidad; es decir, que ambos afectan el rendimiento; por otro lado, el valor $r$ reporta que el $\mathrm{pH}$ fue inversamente proporcional al rendimiento, lo cual coincide con los resultados de Zegada (2015).

Tabla 2. Correlación de Spearman pH, temperatura y rendimiento de la pectina.

\begin{tabular}{ccrc}
\hline & & pH & $\begin{array}{c}\text { TEMPERATURA } \\
\left({ }^{\circ} \mathbf{C}\right)\end{array}$ \\
\hline Rendimiento & $\mathrm{r}$ & $-0,79$ & 0,47 \\
$(\%)$ & $\mathrm{p}$ & 0,00 & 0,01 \\
& $\mathrm{n}$ & 27,00 & 27,00 \\
\hline
\end{tabular}

La tabla 3 compara las características de calidad de la pectina extraída de la cáscara de mango de azúcar maduro frente a una pectina estándar, la humedad de la pectina muestra que la pectina extraída presentó un contenido de humedad por debajo de $10 \%$, el cual es el máximo aceptable por la USP (United States Pharmacopeia 2014) y con respecto a la pectina estándar y a los valores reportados por Orozco y Suárez (2014), se encuentra por debajo; es recomendable un contenido de humedad inferior al $10 \%$, ya que pectinas muy húmedas son difíciles de pulverizar, se adhieren a las superficies, tienen menor estabilidad y tiempo de vida útil, Baltazar et al, (2013). El contenido de cenizas está por debajo de la pectina estándar y del estudio reportado por Cerón y Cardona (2011), lo que se debe probablemente a la composición del suelo 
Tabla 3. Comparación de la caracterización de la pectina extraída de la cáscara de mango de azúcar maduro frente a una pectina comercial (Merck).

\begin{tabular}{lrc}
\hline & $\begin{array}{c}\text { Pectina cáscara de mango } \\
\text { de azúcar maduro }\end{array}$ & Pectina estándar \\
\hline Humedad (\%) & $4,51 \pm 0,807$ & $8,55 \pm 1,96$ \\
Ceniza (\%) & $1,35 \pm 0,074$ & $3,77 \pm 3,39$ \\
Alcalinidad de las cenizas (\% carbonato) & $1,71 \pm 1,136$ & $2,34 \pm 2,90$ \\
Peso equivalente (mg/meq) & $2326,42 \pm 54,12$ & $1775,46 \pm 1143,78$ \\
Acidez libre (meq/g) & $0,86 \pm 0,02$ & $0,78 \pm 0,46$ \\
Contenido de metoxilo (\%) & $11,80 \pm 0,04$ & $6,93 \pm 3,22$ \\
Grado de esterificacion (\%) & $81,69 \pm 0,25$ & $74,71 \pm 3,32$ \\
Aua (\%) & $82,38 \pm 0,17$ & $68,29 \pm 13,39$ \\
Hierro(mg/100g BS) & 25,48 & 9,50 \\
Calcio (mg/ 100 g BS) & 1034,42 & 1006,40 \\
\hline
\end{tabular}

en la que fue cultivado el vegetal, al uso de fertilizantes o a otros factores ambientales que influyen en el contenido de material inorgánico de los vegetales, López et al. (2007).

La alcalinidad de las cenizas obtuvo un valor por debajo de la pectina estándar, pero se relaciona con los resultados obtenidos por Ferreira et al. (1995). La acidez libre se encontró levemente por encima de la pectina estándar, seguramente debido a que la acidez libre aumenta a medida que los $\mathrm{pH}$ son bajos, ya que se desesterifican los grupos carboxilos y su naturaleza se modifica disminuyendo su estado como forma de sales o esteres y aumentando su presencia como grupos ácidos, Cabarcas et al. (2012). El peso equivalente está por encima de la pectina estándar, esto debido a que posiblemente se presentaron gran cantidad de reacciones hidrolíticas del poligacturonano (Arellano y Hernández 2013). El contenido de metoxilo obtenido está por encima de la pectina estándar, y cumple a su vez con lo exigido por USP (United States Pharmacopeia 2014), a partir de este valor la pectina se clasificó de alto metoxilo, esto coincide con el estudio realizado por Chacín et al. (2010).

El porcentaje de esterificación es mayor con respecto a la pectina estándar y se clasifica como de alto grado de esterificación, lo que la influye directamente sobre la capacidad de formar geles (Mamani 2012).

El ácido anhidro galacturónico se encontró por encima de la pectina estándar y cumple con lo exigido por la Farmacopea de los Estados Unidos (70\%), USP (2014), por otro lado se ha establecido que el contenido mínimo de ácido galacturónico para que una pectina sea relevante para la industria alimentaria es del 65\%, USP (2010). El contenido de hierro y de calcio encontrado representa una cantidad superior a la pectina estándar, a su vez se relaciona con los resultados obtenidos por Ferreira et al. 1995, donde prevalece el contenido de calcio, seguido del magnesio y en menor cantidad el hierro.

La mermelada obtenida utilizando la pectina de cáscara de mango de azúcar, tuvo una viscosidad de 9,33 centipoise (cps), un $\mathrm{pH}$ de 2.9 y $65{ }^{\circ}$ Brix, valores que cumplen con lo exigido por la NTC 285 de 2007, ICONTEC (2007), se asemejan a lo reportado por Arellano y Hernández (2013) y con Chaparro et al. (2015), lo que indica que la pectina extraída es adecuada para su utilización en productos que requieren alta gelificación.

Al comparar el espectro de la pectina obtenida de la cáscara del mango de azúcar maduro, con 
respecto al espectro estándar, se evidencian los grupos funcionales característicos de su estructura, los cuales varían levemente con respecto a la pectina estándar.

Se observó que en la banda entre los 3200 y $3600 \mathrm{~cm}^{-1}$ se encuentran los grupos hidroxilo $(-\mathrm{OH})$ demostrando que la pectina es un polisacárido, entre las bandas 2900 y 3000 $\mathrm{cm}^{-1}$ se encuentran vibraciones del grupo alquilo $(\mathrm{CH})$, por otra parte en la banda entre 2000 y $2200 \mathrm{~cm}$ se evidencian unos picos no característicos, indicando que la pectina tenia impurezas, con respecto a la banda entre 1600 y $1800 \mathrm{~cm}$ se encuentran los grupos carbonilos $(\mathrm{C}=\mathrm{O})$ del éster y del ácido, indicando que la pectina es de alto metoxilo, y por último al grupo C-O se encuentran en la banda entre los 1000 y $1100 \mathrm{~cm}$.

\section{CONCLUSIONES}

Las condiciones óptimas de extracción de la pectina fueron: $\mathrm{pH} 1$, temperatura $100{ }^{\circ} \mathrm{C}$, tiempo 60 minutos y se obtuvo un rendimiento promedio del 15,257 $\pm 0,045 \%$ a partir de la cáscara seca.

La composición química de la pectina estudiada determinó valores de peso equivalente de $2326,420 \pm 54,117 \mathrm{~g} \mathrm{mEq}^{-1}$, acidez libre de $0,859 \pm 0,018 \mathrm{~g} / \mathrm{mEq}$, metoxilo de 11,801 \pm 0,036\%, esterificación de 81,688 \pm 0,248\% y contenido de ácido anhidrogalacturónico de $82,380 \pm 0,174 \%$, lo cual la define como una pectina de alto metoxilo, grado de esterificación y de pureza.

\section{REFERENCIAS}

Arellano, G. y Hernández, M. 2013. Evaluación del uso de la pectina extraída del procesamiento de piña y níspero en la preparación de mermeladas. Tesis Ingeniero químico, Universidad Rafael Urdaneta, Maracaibo, Venezuela.
Addosio, R., Páez, G., Marín, M., Marmol, Z. y Ferrer, J. 2005. Obtención y caracterización de la pectina a partir de la cáscara de parchita (Passiflora edulis $\mathrm{f}$. flavicarpa Degener). Revistas Facultad de Agronomía 22:240-249.

Asohofrucol, Ministerio de Agricultura y Desarrollo Rural, Corrpoica, 2009. Descripción de las variedades de mango criollo colombiano. Centro de Investigación Nataima, Tolima, Colombia, p. 9.

Baltazar, R., Carbajal, D., Baca, N. y Salvador, D. 2013. Optimización de las condiciones de extracción de pectina a partir de cáscara de limón Francés (Citrus medica) utilizando la metodología de superficie de respuesta. Revista Agroindustrial Science 3(2): 77-89.

Berardini, N., Carle, R., Knodler, M. y Schieber, A. 2005. Utilization of mango peels as a source of pectin and polyphenolics. Innovative Food Science \& Emerging Technologies 6(4): 442-452.

Cabarcas, E., Guerra, A. y Henao, C. 2012. Extracción y caracterización de pectina a partir de cáscaras de plátano (Hartón Musa AAB simmonds) para desarrollar un diseño general del proceso de producción. Tesis Ingeniero químico, Universidad de Cartagena, Colombia.

Campos, M. y Guerrero, A. 2014. Estimación teórica del proceso de transformación del mango (Manguifera indica L.) y aprovechamiento de sus subproductos en los municipios de Chicoral, Espinal y Gualanday, departamento del Tolima. Tesis Ingeniero de alimentos, Universidad Nacional Abierta y a Distancia (UNAD), Ibagué, Colombia.

Chacín, J., D’addosio, R. y Marín, M. 2010. Evaluación del contenido de pectina en diferentes genotipos de guayaba de la zona sur del Lago de Maracaibo, Multiciencias, 10(1): $7-12$. 
Chaparro, S., Marquez, R., Sánchez, J., Vargas, M. y Gil, J. 2015. Extracción de pectina de fruto del higo (Opuntia Ficus Indica) y su aplicación en un dulce de piña, Revista U.D.C.A. Actualidad \& Divulgación Científica, 18(2): 435-443.

Cerón, I. y Cardona, C. 2011. Evaluación del proceso integral para la obtención de aceite esencial y pectina a partir de cáscara de naranja, Ingeniería y Ciencia, 7(13): 65-86.

Dussan, S., Torres, C y Reyes, P. 2014. Efecto del recubrimiento comestible sobre los atributos físico-químicos de mango Tommy Atkins mínimamente procesado y refrigerado, Acta Agronómica, 63(3): $1-18$.

Falcon, M., Barrón, J., Romero, Ana y Domínguez, M. 2011. Efecto adverso en la calidad proteica de los alimentos de dieta con alto contenido de fibra dietaria, Revista Chilena de Nutrición, 38(3): 369 375.

Fennema, O. 1993. Química de los alimentos. Acribia, Zaragoza, España, p 1258.

Ferreira, S. 2007. Pectinas: aislamiento, caracterización y producción, editorial Universidad nacional de Colombia. $\mathrm{p}$ 186

Ferreira, S., Peralta, A y Rodríguez, G. 1995. Obtención y caracterización de pectina a partir de desechos industriales del mango (cáscara), Revista Colombiana de Ciencias Químico Farmacéuticas, 24(1): 29-34.

Flórez, C. 2013. Elaboración y evaluación nutricional comparativa de mermelada de guayaba (Psidium guajava) deshidratada frente a mermeladas casera e industrial. Tesis Bioquímico farmacéutico, Escuela Superior Politécnica de Chimborazo, Riobamba, Ecuador.
Gamboa, M. 2009. Aprovechamiento de los residuos obtenidos del proceso de despulpado del mango (Mangifera indica L.), de las variedades Smith, Tommy Atkins, Haden y Bocado como materias primas para la obtención de pectinas. Tesis Magíster en Ciencias de los Alimentos, Universidad de Oriente, Puerto La Cruz, Venezuela.

García, M., López, L. y Rodríguez, L. 2010. Determinación de la composición química y actividad antioxidante en distintos estados de madurez de frutas de consumo habitual en Colombia, mora (Rubus glaucus B.), maracuyá (Passiflora edulis S.), guayaba (Psidium guajava L.) y papayuela (Carica cundinamarcensis J.). Revista Alimentos Hoy, 19(21): 35-42.

García, C. y Penagos, C. 2011. El entorno comercial de la pectina en la industria alimentaria antioqueña, Revista Soluciones de Postgrado EIA. (7):121-131.

Instituto Colombiano de Normas Técnicas y Certificación. 2002. Norma Técnica Colombiana (NTC) 5139: Frutas frescas: Mangos criollos, Bogotá, D.C., 22 p.

Instituto Colombiano de Normas Técnicas y Certificación. 2007. Norma Técnica Colombiana (NTC) 285: Frutas procesadas, mermeladas y jaleas de frutas. Bogotá, D.C., 8 p.

Koubala, B., Kansci, G., Mbome, L. Crépeau, M., Thibault, J., Ralet M. 2008. Effect of extraction conditions on some physicochemical characteristics of pectins from "Améliorée" and "Mango" mango peels. Revista FoodHydrocolloids, 22(7): 1345-1351.

León, D. y Riveros, J. 2014. Extracción y caracterización química de las pectinas de las cáscaras del maracuyá amarillo (Passiflora edulis, Var flavicarpa degener), granadilla (Passiflora ligularis Juss) y tumbo serrano (Passiflora mollísima H.B.K. Bailey). Tesis Ingeniero Químico, Universidad Nacional Del Callao, Perú. 
López, P., Prieto, F., Gaytán, M y Román, A. 2007. Caracterización fisicoquímica de diferentes variedades de cebadas cultivadas en la región centro de México. Revista Chilena de Nutrición, 34(1): 71-77.

Mamani, P., Ruíz, R. y Veiga, M. 2012. Pectina: Usos Farmacéuticos y Aplicaciones Terapéuticas. Anales de la Real Academia de Farmacia, Madrid, España 78(1): 82-97.

Martínez, M., Ortiz, B., Pérez, C. y Anzola, C. 2011. Efecto de la pectina extraída de guayaba sobre el perfil lipídico en adultos con diferente condición cardiovascular. Revista de la Facultad de Medicina, 59(2): 103-111.

Medina, J. 1981. Frutas tropicais: manga. Alguns aspectos tecnológicos das frutas tropicais e seus productos. Instituto de Tecnología de Alimentos, Sao Pulo, Brasil, p. 296.

Montaña, K. 2016. Obtención de pectina a partir de badea (Passiflora quadrangularis). Tesis en Maestría en Ciencias Químicas, Universidad Nacional de Colombia, Bogotá D.C.

Morton, J. 1987. Fruits of warm climates. Creative Resource Systems, Miami, 221239.

Muñoz, F. 2011. Extracción y caracterización de la pectina obtenida a partir del fruto de dos ecotipos de cocona (Solanum sessiliflorum), en diferentes grados de madurez; a nivel de planta piloto. Tesis Maestría en ingeniería agrícola, Universidad Nacional de Colombia, Bogotá D.C.
Orozco, D. y Suárez, D. 2014. Obtención y caracterización de pectina a partir de la cascarilla de cacao (Theobroma cacao L.), subproducto de una industria chocolatera nacional. Tesis Químico industrial, Universidad Tecnológica de Pereira, Pereira, Colombia.

Sriamornsak, P. 2003. Chemistry of Pectin and Its Pharmaceutical Uses: A Review, Silpakorn University International Journal. 3(2): 206-208

Suzanne, S. 2010. Viscosity Measurement using a brookfield viscometer. En: Food Analysis Laboratory, Manual 2. Editorial Springer, New York, p $69-72$.

Stuart, B. 2005. Infrared Spectroscopy: Fundamentals and Applications. John Wiley \& Sons Ltd, England, p 208.

The United States Pharmacopeial Convention. 2014. Formulario nacional. USP $37, \mathrm{NF}$ 32, TwinbrookParkway, Rockville, MD, Estados Unidos de América , p 4786 $-4787$.

The United States Pharmacopeial Convention. 2010. Food Chemicals Codex Monographs, United States.

Zegada, V. 2015. Extracción de pectina de residuos de cáscara de naranja por hidrólisis ácida asistida por microondas (HMO). Revista UPB Investigación \& Desarrollo, 15(1): 65-77. 